\title{
The relationship between $2 d$ knee valgus angle during single leg squat (SLS), single leg landing (SLL), and forward running.
}

\author{
Atkin, K., Herrington, L.C., Alenezi, F.S., Jones, P.A. and Jones, R. \\ University of Salford
}

Background: Two-dimensional (2D) analysis of knee valgus during common athletic screening tasks such as SLS has been purported to identify individuals who may be at a high-risk of ACL injury. There is limited literature exploring the relationships between joint motion during SLS and other athletic tasks associated with knee joint injuries, in order to evaluate the effectiveness of the SLS to identify athletes with hazardous knee motion in a range of athletic tasks.

Objective: To assess the relationship between the 2D knee valgus angle among three tasks (SLS, SLL, and Running).

Design: A correlational study.

Setting: Undertaken in the Human Performance Laboratory at the University of Salford.

Participants: 15 recreational athletes, 7 males and 8 females, were recruited (age $25.2 \pm 5.1$ years; height $1.6 \pm 7.38 \mathrm{~m}$; and mass $67.6 \pm 10.93 \mathrm{~kg}$ ).

Intervention: Participants performed a series of SLS, SLL from a 31-cm step, and forward running at percieved maximum speed whilst being videotaped with a 2D digital camera.

Main outcome measurements: Maximum right knee valgus angle was quantified by measuring the frontal plane projection angle (FPPA) using Quintic Biomechanics v21 software package.

Results: A moderate correlation was shown between FPPA during SLS and SLL when the whole participant group was analysed $(r=0.35)$. When split by gender female statistics showed good correlations between the majority of tasks; SLS and SLL ( $r=0.87)$, SLS and Run ( $r=0.59)$ but weaker between SLL and Run (0.26).

Conclusions: In females the FPPA during SLS correlates with FPPA during SLL and running. This indicates that if a female patient has increased FPPA during SLS they are likely to have increased FPPA across all the tasks. This could potentially reduce the time and tasks required for screening, as only one task would need to be assessed. In males there is little correlation between tasks so the same would not apply in male subjects. 